\title{
Metodología para el Diagnóstico de Prácticas del Modelo Proceso Personal de Software
}

\author{
Plinio Puello, Stalin D. Oviedo y David A. Franco \\ Universidad de Cartagena, Facultad de Ingeniería, Grupo de Investigación en Soluciones Tecnológicas de \\ Nueva Generación para el Desarrollo Social, Industrial y Empresarial, E-SOLUCIONES, \\ Avenida del Consulado, Calle 30, No. 48 - 152, Cartagena-Colombia. \\ (e-mail: ppuellom@unicartagena.edu.co, stalin.oviedo@gmail.com,dfrancob@unicartagena.edu.co).
}

Recibido Jul. 31, 2013; Aceptado Sep. 27, 2013; Versión final recibida Nov. 28, 2013

\begin{abstract}
Resumen
El objetivo principal de este trabajo fue desarrollar una metodología para el diagnóstico de prácticas del Proceso Personal de Software en las empresas de Cartagena de Indias, Colombia. Para ello se utilizó variables relacionadas con el proceso de desarrollo, fundamentado en una metodología con enfoque sistémico para la verificación del conocimiento y uso del modelo de Humphrey, donde cada subsistema actuó con entradas y salidas hacia los otros. Esta investigación propende mejorar las prácticas del proceso personal de software dando el primer paso en un camino hacia la calidad requerida y detectada en una investigación previa presentada en la literatura. Los resultados obtenidos al realizar el diagnóstico evidenciaron buenas prácticas en empresas con metodologías definidas para el desarrollo personal del producto software contrario a otras que carecían de estándares para el manejo de rutinas preestablecidas.
\end{abstract}

Palabras clave: proceso personal de software, diagnóstico, desarrollo de software, calidad de software, metodología.

\section{Methodology for the Diagnosis of Practice Model Personal Software Process}

\begin{abstract}
The aim of this paper was to develop a methodology to diagnose the use of personal software process in software industry of Cartagena de Indias, Colombia. In this work, Personal Software Process related model based on a systemic methodology variables were used taking as reference the Humphrey's model in which each subsystem had input and output variables to other subsystems. This work tends to improve the techniques of Personal Software Process taking the first step on a path to the required quality and detected in a previous work presented in the literature. The results showed good practice in companies with defined methodologies for software development in contrast to others who lacked standards for managing of established routines.
\end{abstract}

Keywords: personal software process, diagnosis, software development, software quality, methodology. 


\section{INTRODUCCIÓN}

El desarrollo de una metodología para el diagnóstico de las prácticas del modelo PSP (Personal Software Process) (Humphrey, W. S.,1996), foco principal de esta investigación, hace parte del proceso de mejoramiento académico y de conocimiento del entorno empresarial que se propende desde el interior de la asignatura electiva de noveno semestre del Programa de Ingeniería de Sistemas de la Universidad de Cartagena, Gestión de la Calidad - Mantenimiento y Pruebas de Software, donde el docente que la imparte, el cual es autor del presente artículo, investiga sobre el conocimiento y uso de modelos de calidad para mejorar la competitividad del sector del desarrollo de software en la ciudad de Cartagena de Indias Colombia.

Siendo el primer paso hacia la calidad del producto, el proceso de desarrollo personal de software y pilar para la implementación de certificaciones de otros estándares de calidad representa el objetivo principal de esta investigación a través del planteamiento de una metodología para el diagnóstico de prácticas del Modelo PSP.

El presente trabajo se formula con el interrogante, ¿Cómo los desarrolladores de software de las empresas planean y organizan su trabajo de desarrollo, buscando determinar el estado actual de las buenas prácticas del proceso de desarrollo personal? Resultante de lo anterior se generó una propuesta metodológica para realizar el diagnóstico de las rutinas utilizadas por el personal que labora de las empresas buscando la mejora del proceso aumentando la previsibilidad y eficacia. Otras investigaciones relacionadas con PSP aplican un instrumento de recolección de información con el objetivo de medir o registrar los efectos de la aplicación de la metodología PSP como herramienta en la enseñanza de los cursos de programación (Cardona et al., 2012; Wohlin, C., y Wesslén, A., 1998).

Existen otras investigaciones que buscan establecer los roles y hábitos disciplinares, a partir de una estrategia en la que se aplican metodologías PSP y TSP en el proceso de formación del ingeniero informático, durante los distintos años de la carrera (López et al., 2011) o desarrollo de software de escritorio (Salinas et al., 2011; Silliti et al., 2003) o web (Syu et al., 1997) para el soporte de recolección de datos de los modelos mencionados, debido al problema de la calidad de los datos recolectados (Johnson, P. M., y Disney, A. M. 1999; Sison, R., 2005; Kemerer, C. F., y Paulk, M. C., 2009);se puede determinar que las investigaciones anteriores se realizaron para un entorno académico. Sin embargo, el trabajo reseñado en este artículo tiene aplicabilidad empresarial al igual que los presentados por otros autores (Morisio, M., 2000; Robert L. C., 1999), o también con el objeto de determinar la relación costo beneficio del modelo al aplicarlo (Lee et al., 2008; Silberberg, D., 1998).

En otra investigación realizada en la Universidad de Cartagena, Colombia, se encontraron carencias en las empresas desarrolladoras de software, cuyo objetivo fue describir las tendencias acerca del uso de los Modelos de Calidad Software en las empresas desarrolladoras de Software de la ciudad de Cartagena realizando un estudio acerca del conocimiento y utilización de estos modelos (Buendía, 2011). Se obtuvo, a través de un instrumento de 42 preguntas, la medición de variables como: conocimiento de los modelos de calidad, herramientas utilizadas para evaluar la calidad, documentación de los productos, niveles de competitividad e impacto de los clientes en el desarrollo de software. Entre otros resultados se obtuvo que solo dos (2) empresas de dieciocho (18) encuestadas conocían acerca del modelo PSP y TSP. Además, cuatro (4) de estas identificaban el modelo CMMI y que la mayoría de las ellas no seguían un modelo definido sino adaptado a las particularidades e intereses de cada una.

Algunas de las conclusiones de la investigación anterior fue la falta de claridad en los procesos de desarrollo y de calidad a nivel interno, justificando la continuidad de este tema con la propuesta metodológica para el diagnóstico de las prácticas del modelo PSP, ya que al implementar este modelo en las empresas desarrolladoras de software se obtendrán datos históricos evaluando las rutinas preestablecidas buscando ser más previsibles y eficaces. Siguiendo en un proceso de mejoramiento con el cumplimiento de los modelos TSP y CMMI aumentando la calidad en todas las áreas de la empresa.

La metodología propuesta fue desarrollada en cinco (5) fases considerando: La definición de las variables asociadas al modelo PSP, la construcción de los instrumentos de recolección de información con base en estas variables, el proceso de recolección de la información, la ponderación de los resultados sobre las variables y el diagnóstico de forma global y por variables para las empresas.

\section{METODOLOGÍA PARA EL DIAGNÓSTICO DE PRÁCTICAS DEL MODELO PSP}

La metodología para la realización del diagnóstico de las prácticas del modelo PSP a nivel empresarial, propuesta en este artículo, especifica cinco (5) fases en las cuales el cumplimiento de las primeras cuatro 
(4) proporcionan una base de información necesaria para la realización del diagnóstico, siendo la última fase la recopiladora y emisora de este, a continuación se describen cada una de las cinco (5) fases:

Fase 1: Definición de las variables asociadas al modelo PSP

Las variables definidas con relación al modelo PSP fueron enfocadas al empleo de las prácticas o actividades presentes en el flujo de procesos del modelo (Humphrey, W. S., 2005), como se puede apreciar en la figura 1.

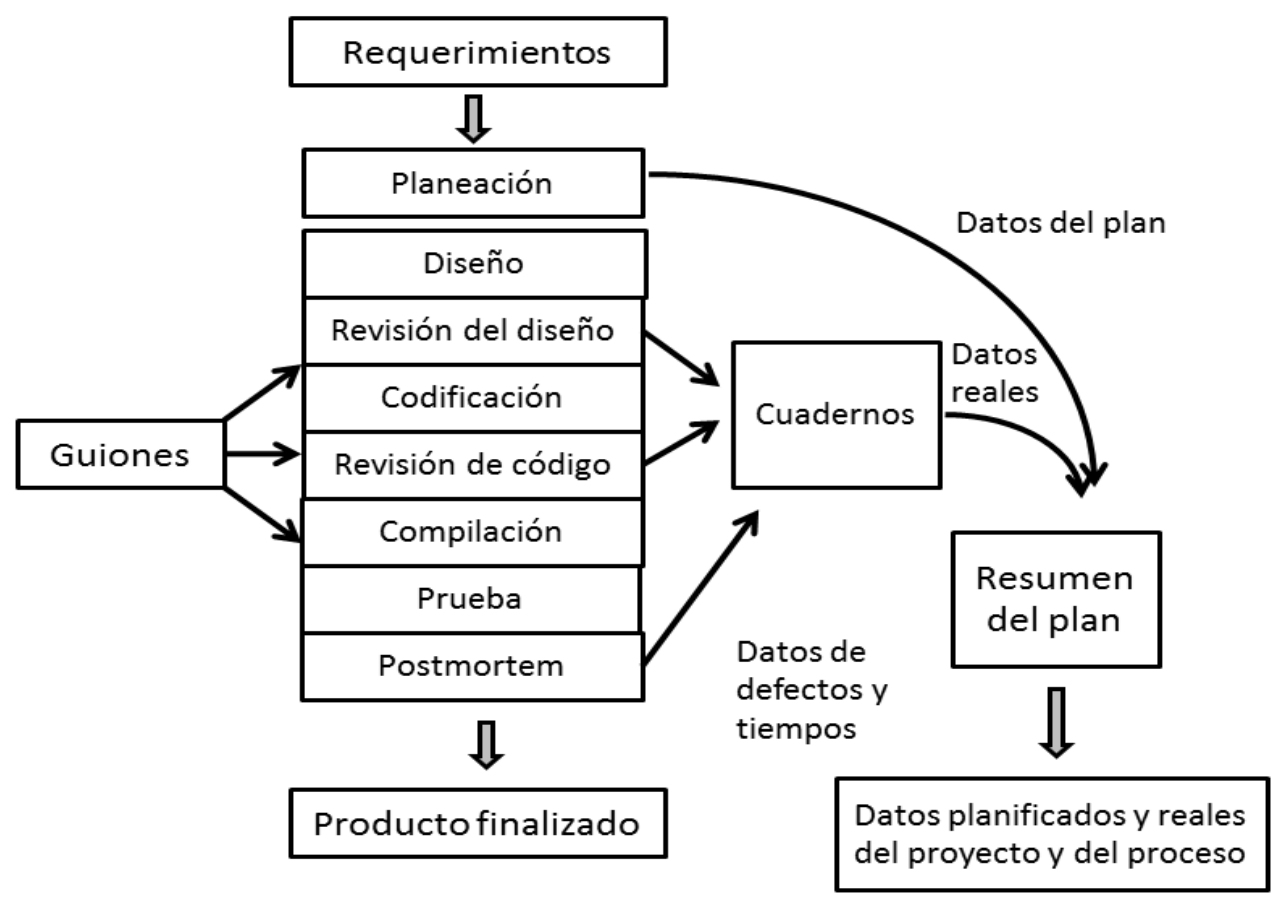

Fig. 1: Flujo de procesos del Modelo PSP (Humphrey, W. S., 2005).

El proceso para definir las variables no siguió una metodología de tipo experimental o cuasi-experimental como lo mencionan algunos autores (Seaman, 1999; Kitchenhamet al., 2002; Kampenes et al., 2009). Sin embargo, a través del enfoque sistémico (Bertalanffy, 1968; Capra, 1998; Chiavenato 1999) se definieron las variables para medir el conocimiento y uso del modelo PSP en las empresas, estableciendo las relaciones del flujo de procesos entre subsistemas que gestionan y vuelven viable el modelo. Para el planteamiento de las preguntas y opciones de respuesta se tuvo en cuenta el cumplimiento y verificación del sistema compuesto por subsistemas (procesos) del modelo PSP, siguiendo lo expuesto por los anteriores autores y las partes que lo conforman evidenciado en la figura 1, en la cual se aprecian las variables distribuidas de la siguiente manera:

Manejo de los requerimientos: Esta variable es de tipo cuantitativa porcentual y mide el nivel de manejo de los requerimientos, que son la parte inicial del proceso PSP. La salida de este proceso servirá de entradas a otros relacionados con la planeación.

Realización de planes: Esta variable es de tipo cuantitativa porcentual y mide el nivel de realización de los planes de proyecto a nivel personal y que según la figura 1, es el segundo paso del flujo de procesos del modelo. La salida de este proceso permite verificar el cumplimiento en el resumen del plan y es la entrada de otros subsistemas (procesos) como diseño y medición del tiempo de desarrollo.

Empleo de buenas prácticas o herramientas para el modelado: Esta variable es cuantitativa porcentual y describe el uso de buenas prácticas para el modelado, bien sea UML u otras, en la figura 1 se refleja en el subsistema de diseño (proceso) relacionados con otros subsistemas como planeación, requerimientos, revisión del diseño, codificación y revisión del código.

Estimación del tamaño del producto: Esta variable es cuantitativa porcentual y mide el nivel de estimación del desarrollador para las actividades asignadas. Aunque esta variable no se refleja directamente en la figura 1, está contenida en el libro Introducción al Proceso Software Personal (Humphrey, 2001), y relevante su consideración por su relación con otros subsistemas (procesos) de la figura anterior. 
Medición del tiempo de desarrollo: Esta variable es cuantitativa porcentual y mide el nivel de medición del tiempo de desarrollo, en la figura 1 se refleja bajo el enfoque sistémico como una variable auxiliar con los cuadernos de trabajo donde se recolecta información de los otros subsistemas (procesos).

Manejo de defectos: Esta variable es cuantitativa porcentual y mide el nivel de manejo de los defectos introducidos y corregidos durante el proceso de desarrollo de software, en la figura 1 se refleja en cualquiera de los procesos posteriores a la planificación y los cuadernos de trabajo. Esta variable posee el mayor número de preguntas en el instrumento propuesto por el nivel de profundización descrito por Humphrey (2001) y por la relación de la misma con otros subsistemas (procesos) como: Revisión del diseño, codificación, Revisión del código, compilación y pruebas.

Recolección de datos y realización de reportes: Esta variable es cuantitativa porcentual y describe el nivel de realización del proceso de recolección de datos y formulación de reportes, evidenciando el uso de la metodología PROBE, en la figura 1 se refleja con el uso que se le da a los cuadernos de trabajo como variable auxiliar donde se recolectan los datos.

El empleo de estas variables cumplió con los requerimientos estimados desde el punto de vista sistémico cumpliendo con la dinámica de los subsistemas (procesos) descritos en la figura 1 para la medición de las prácticas del modelo PSP: Lo anterior, ayudó a plantear las preguntas con opciones de respuesta cuantitativas porcentuales.

\section{Fase 2: Construcción de los instrumentos de recolección de información con base en las variables}

Se establecieron cuatro (4) opciones de respuesta a las preguntas de las encuestas de PSP, tres (3) de ellas fueron opciones definidas y una opción "otra" para el caso en que la respuesta del personal encuestado en las empresas no se considerara en las tres (3) anteriores, en ciertos casos eran preguntas cerradas y otras de justificación o explicación. De las tres (3) opciones de respuesta definidas siempre existió una que de ser escogida, demostraba el uso de las mejores prácticas.

La encuesta de PSP se conformó por un número total de quince (15) preguntas con una extensión de tres (3) páginas y una duración de cinco (5) a diez (10) minutos, para su diligenciamiento, siendo una encuesta relativamente corta que obtuvo gran aceptación entre las nueve (9) empresas que mostraron interés en participar en la investigación. Las preguntas y opciones de respuestas que constituyen este instrumento de recolección de información son las siguientes:

Variable: Manejo de los requerimientos

¿De qué forma establece los requerimientos del software?

Opciones de respuesta

Hago una conclusión personal de lo que quiere el cliente.

Hablo con el cliente solo una vez para determinar sus necesidades.

Obtengo una lista de requerimientos que me es asignada por un ingeniero de requerimientos

Otra

¿Cómo considera el entendimiento de los requerimientos de lo que va a desarrollar?

Opciones de respuesta

Me cuesta trabajo comprender lo que realmente quiere el cliente o los requerimientos que me son asignados

Entiendo los requerimientos luego de revisarlos muchas veces con el cliente

Estoy conectado con los requerimientos del cliente y entiendo su necesidad sin ningún problema

Otra

Variable: Realización de planes

¿Efectúa previamente a la realización de un programa o módulo software, una planeación u organización de las actividades a realizar?

Opciones de respuesta

Síno

¿Cómo realiza la planeación de su trabajo asignado?

Opciones de respuesta

Establezco mentalmente algunos objetivos y luego me pongo a desarrollar.

Documento una lista de tareas algo detallada y luego las cumplo una por una

Planeo usando un SCRIPT de trabajo que me guiará en el proceso de desarrollo de forma detallada

Otra 
Variable: Estimación del tamaño del producto

¿Realiza una estimación del tamaño del producto a desarrollar al analizar los requerimientos?

Opciones de respuesta

SíNo

¿En que se basa para la estimación del tamaño de producto?

Opciones de respuesta

En mi experiencia personal como programador

Me es difícil determinar el tamaño, debido a que no conozco algún criterio para la estimación.

Utilizo la metodología PROBE para la estimación de tamaño

Otra

Variable: Medición del tiempo de desarrollo

¿Mide constantemente el tiempo empleado en cada uno de sus procesos de desarrollo?

Opciones de respuesta

Síno

¿Cómo logra hacer estas mediciones?

Opciones de respuesta

Basado en mi experiencia personal.

Basado en un historial de proyectos realizados y tomando una estimación personal

Utilizando una estimación de recursos por medio de la metodología PROBE

Otra

Variable: Manejo de defectos

En cuanto a los defectos en el software, usted:

Opciones de respuesta

Considera que solo aparecen en la codificación y solo es necesario corregirlos en la fase de pruebas

Considera que pueden aparecer en cualquier fase del desarrollo del software y los corrige en la fase de pruebas

Considera que pueden aparecer en cualquier fase del desarrollo del software y los corrige inmediatamente.

Otra

Considera que para eliminar los defectos de un software lo mejor es:

Opciones de respuesta

Corregirlos al final de cada fase

Corregirlos en las fases más tempranas del proyecto

Tratar de prevenir la aparición de estos, utilizando buenas prácticas.

Otra

¿Documenta o registra de manera controlada los defectos y modificaciones que surgen en el desarrollo?

Opciones de respuesta

SiNo

¿De qué forma realiza esta documentación?

Opciones de respuesta

Guardo mentalmente la información y la comunico a quien la solicite.

Creo un documento personal más o menos detallado para la consignación de esta información

Utilizo formatos especiales para la recopilación de esta información en cada fase de la planeación.

Otra

Variable: Recolección de datos y realización de reportes

En cuanto a las líneas de código del programa, usted:

Opciones de respuesta

Cree innecesario contarlas ya que no representan un dato relevante en el desarrollo del programa.

Cree necesario contarlas, pero no conoce algún método ágil y preciso para el conteo.

Utiliza estándares precisos para el conteo de líneas de código y documenta la información del conteo en formatos especiales

Otra

Cuando finaliza una tarea que le es asignada, usted:

Opciones de respuesta

Espera a su próxima asignación 
Llena un formato de resumen con toda la información registrada en cada etapa del proceso

Tiene en cuenta mentalmente observaciones del trabajo y las comunica a quien sea necesario

Otra

Variable: Empleo de buenas prácticas o herramientas para el modelado

¿Qué herramientas o procedimientos utiliza en la fase de diseño?

Opciones de respuesta

No realizo ningún diseño de lo que voy a desarrollar

Realizo diseños empleando diagramas UML, pero por lo general obtengo una codificación que no concuerda mucho con ellos

Realizo diseños empleando diagramas UML, aplicando patrones de ingeniería de software y obtengo una buena codificación

Otra

\section{Fase 3: Proceso de recolección de la información}

Para el proceso de recolección de información se realizó en primera instancia una selección de las empresas que participarían en la investigación, esto se llevó a cabo con una muestra de empresas desarrolladoras de software de la ciudad de Cartagena de Indias, por lo que se le solicitó a la Cámara de Comercio de Cartagena, una lista de empresas con un objeto social relacionado al desarrollo y la comercialización de software. En total se identificaron ciento diecisiete (117) empresas de las cuales en su mayoría eran unipersonales y de poco personal, para lo cual en los objetivos de esta investigación, solo fueron seleccionadas aquellas con el siguiente perfil:

Un número de empleados de mínimo 3 personas.

Una actividad económica relacionada al desarrollo de software.

Patrimonio mayor a $\$ 1.000 .000$ de pesos colombianos.

Un tiempo en el mercado de más de 2 años.

El número de empleados es importante porque permite validar la no unipersonalidad de la empresa, la actividad económica garantizó que se realizara un estudio a las organizaciones con el objeto social correcto debido a que en la lista aparecieron empresas con actividades económicas poco relacionadas al desarrollo de software. La liquidez económica es un factor importante, debido al costo de implementación de los modelos de calidad, no tiene sentido tomar empresas con poco capital para implementar modelos de alto costo y que requieren de un tamaño organizacional considerable. Finalmente, la presencia en el mercado de más de 2 años permite considerar a la empresa como estable en el cumplimiento del objeto social.

Al aplicar el filtro anterior fueron excluidas el $82.9 \%$ de estas, quedando un total de veinte (20) empresas que representaron la población. Para el cálculo de la muestra con una población conocida y pequeña, con un 95\% de confianza y un 3\% de margen de error, se aplicó la ecuación (1) (Vallejo, 2012):

$n=\frac{N}{1+\frac{e^{2}(N-1)}{z^{2} p q}}$

Donde $\mathrm{n}$ es el tamaño de la muestra, $\mathrm{N}$ es el tamaño de la población, e es el margen de error del $3 \%, \mathrm{z}$ representa el nivel de confianza del $95 \%$, con un valor de 1.96, pq representa la varianza de la población que se maneja con el valor de 0.25 . Dando como resultado una muestra de 19 empresas a las cuales se les invitó a participar en esta investigación por medio de llamadas telefónicas y visitas a las mismas, a lo que contestaron afirmativamente un total de nueve (9) que representaron la muestra final. Dado que diez (10) empresarios no se vincularon a la investigación, se sustenta la selección final no probabilística de acuerdo a Fernández(2004) a través del muestreo por conveniencia el cual consiste en seleccionar unidades más convenientes para el estudio o en permitir que la muestra que participa sea totalmente voluntaria, dando validez al resultado obtenido.

\section{Fase 4: Ponderación de los resultados sobre las variables}

La ponderación radica en la valoración de las variables evaluadas con el instrumento de recolección de información, realizando al final una ponderación global de cada modelo respectivo para cada una de las empresas encuestadas, consistiendo en la suma de las valoraciones ponderadas de las distintas variables de la siguiente manera: 
El modelo de diagnóstico para PSP, fue construido con la herramienta ofimática Excel, generando una hoja de cálculo en la que se organizaron las variables con sus respectivas preguntas, a las cuales en sus respuestas se le asignaron valores numéricos de uno (1) a tres (3) con el objetivo de ponderar (Valoración cuantitativa) las respuestas en cuanto al nivel de conocimiento o empleo de buenas prácticas. La ponderación se realizó de la siguiente forma:

Ponderación baja: esta tiene un valor numérico de uno (1) y describe la forma menos eficiente de responder o evidenciar buenas prácticas.

Ponderación media: esta tiene un valor numérico de dos (2) y describe una forma medianamente eficiente de responder o evidenciar buenas prácticas.

Ponderación alta: esta tiene un valor numérico de tres (3) y describe la mejor forma de responder o evidenciar buenas prácticas.

Lo anterior aplicó a aquellas preguntas de cuatro (4) opciones de respuestas, con tres (3) definidas y una (1) opción con la descripción "otra", a la cual se le asigna un valor de uno (1) a tres (3), dependiendo de las consideraciones del evaluador o evidencia que se le presente. Este tipo de ponderación afecta de manera directa la valoración de la variable ya que hacen parte del total de puntos asignados a esta misma.

Las preguntas cerradas en sus respuestas recibieron una ponderación de uno (1) a cero (0), afectan indirectamente la valoración de la variable ya que si bien sus valores asignados no hicieron parte del total de puntos asignados a la variable, condicionaron la otorgación de puntos de preguntas con cuatro (4) opciones de respuesta. Así, si la respuesta es no, no se obtienen puntos por la siguiente pregunta relacionada.

\section{Fase 5: Diagnóstico global y por variables de las empresas participantes}

Con la información proporcionada por cada empresa y aplicando la ponderación de las variables definidas del modelo se prosiguió a establecer dos tipos de diagnóstico por cada empresa los cuales fueron los siguientes:

Diagnóstico PSP por variables: Se expresó el porcentaje de cumplimiento de cada una de las variables evaluadas, por medio de una tabla de valores con rango de $0 \%$ a $100 \%$, correspondiendo al menor y mayor cumplimiento posible del modelo PSP, respectivamente. Las variables evaluadas son:

Variable 1: Manejo de los requerimientos

Variable 2: Realización de planes

Variable 3: Estimación del tamaño del producto

Variable 4: Medición del tiempo de desarrollo

Variable 5: Manejo de defectos

Variable 6: Recolección de datos y realización de reportes

Variable 7: Empleo de buenas prácticas o herramientas para el modelado

La siguiente tabla muestra el diagnóstico de las empresas comparadas por variables. En este caso, y por razones de confidencialidad concertadas con los empresarios, los nombres de las empresas se mostrarán con letras de la A hasta la I como se aprecia en la tabla 1.

Tabla 1: Diagnóstico PSP por variables

\begin{tabular}{|c|c|c|c|c|c|c|c|c|}
\cline { 2 - 8 } \multicolumn{1}{c|}{} & Variable 1 & Variable 2 & Variable 3 & Variable 4 & Variable 5 & Variable 6 & Variable 7 & $\begin{array}{c}\text { Resultado } \\
\text { Global }\end{array}$ \\
\hline Empresa A & $66.67 \%$ & $0.00 \%$ & $33.33 \%$ & $33.33 \%$ & $66.67 \%$ & $50.00 \%$ & $66.67 \%$ & $51.52 \%$ \\
\hline Empresa B & $83.33 \%$ & $33.33 \%$ & $33.33 \%$ & $0.00 \%$ & $66.67 \%$ & $33.33 \%$ & $100.00 \%$ & $54.55 \%$ \\
\hline Empresa C & $83.33 \%$ & $66.67 \%$ & $33.33 \%$ & $33.33 \%$ & $77.78 \%$ & $33.33 \%$ & $66.67 \%$ & $60.61 \%$ \\
\hline Empresa D & $66.67 \%$ & $66.67 \%$ & $33.33 \%$ & $66.67 \%$ & $88.89 \%$ & $33.33 \%$ & $33.33 \%$ & $60.61 \%$ \\
\hline Empresa E & $83.33 \%$ & $66.67 \%$ & $33.33 \%$ & $66.67 \%$ & $66.67 \%$ & $50.00 \%$ & $100.00 \%$ & $66.67 \%$ \\
\hline Empresa F & $83.33 \%$ & $66.67 \%$ & $66.67 \%$ & $66.67 \%$ & $100.00 \%$ & $66.67 \%$ & $100.00 \%$ & $81.82 \%$ \\
\hline Empresa G & $100.00 \%$ & $66.67 \%$ & $66.67 \%$ & $66.67 \%$ & $77.78 \%$ & $83.33 \%$ & $100.00 \%$ & $81.82 \%$ \\
\hline Empresa H & $100.00 \%$ & $100.00 \%$ & $33.33 \%$ & $66.67 \%$ & $100.00 \%$ & $66.67 \%$ & $100.00 \%$ & $84.85 \%$ \\
\hline Empresa I & $100.00 \%$ & $100.00 \%$ & $100.00 \%$ & $100.00 \%$ & $88.89 \%$ & $100.00 \%$ & $100.00 \%$ & $96.97 \%$ \\
\hline
\end{tabular}


Las empresas A y B poseen valores de $0 \%$ en dos variables siendo éstas la del menor resultado en el diagnóstico global (ver Figura 2). Las empresas C, D y E poseen variables con valores superiores al $33,33 \%$ hasta $100 \%$ llegando con diagnósticos globales medios oscilando entre $60 \%$ y $80 \%$. Y finalmente, las empresas F,G,H e I poseen variables con valores de cumplimiento superiores al $66,66 \%$ en su mayoría hasta el $100 \%$ obteniendo un diagnóstico global correspondiente con los resultados de las variables, es decir, superiores al $80 \%$.

Diagnóstico global por empresa: Expresa el porcentaje de cumplimiento del modelo de calidad, construyéndose a partir de la suma de las ponderaciones hechas en los diagnósticos por variables.

En la figura 2 se muestran los resultados de diagnóstico global identificando a las empresas $\mathrm{F}, \mathrm{G}, \mathrm{H}$ e I como las que cumplen en mayor nivel las prácticas exigidas por las variables del Modelo PSP y por lo tanto se evidencia un alto cumplimiento con valores superiores al $80 \%$.

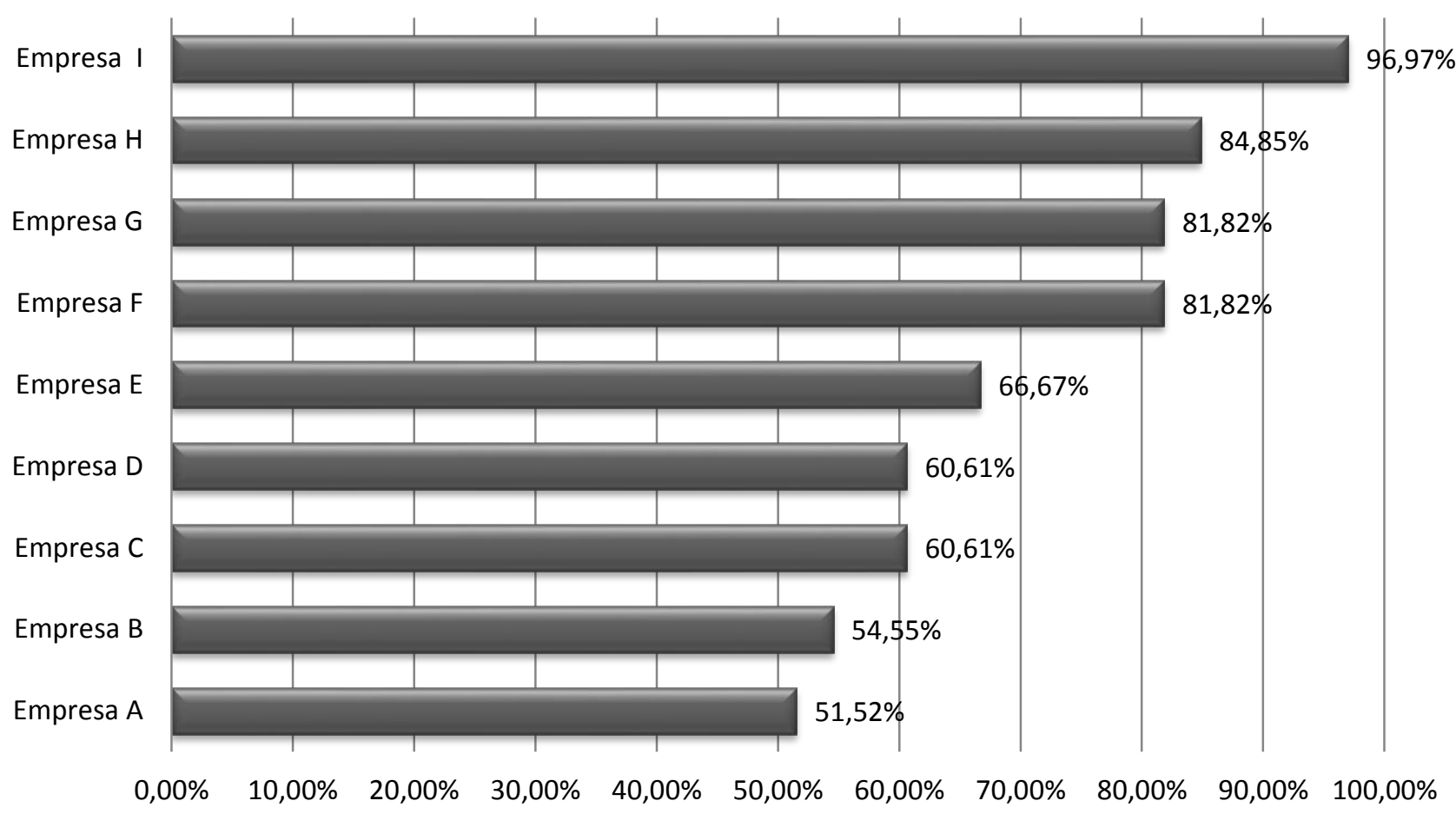

Fig. 2: Diagnóstico global por empresa

Las demás empresas de la figura anterior, deben mejorar sus procesos en el desarrollo de software para alcanzar resultados más altos en las prácticas del Modelo PSP, siendo éste la base fundamental en el proceso de certificación TSP (Team Software Process) y posteriormente CMMI (Capability Maturity Model Integration).

\section{CONCLUSIONES}

Este artículo presentó el desarrollo de una metodología para el diagnóstico de las prácticas del modelo PSP, y gracias a la participación de nueve (9) empresas desarrolladoras de software de la ciudad de Cartagena de Indias, fue posible la aplicación de esta, permitiendo verificar su efectividad y establecer diversos diagnósticos en una muestra de empresas con renombre y solidez en el medio cartagenero, con esto se puede concluir lo siguiente:

1) La metodología permite registrar un estado de calidad de una organización en cuanto a prácticas del modelo PSP;

2) Aquellas empresas que se encuentran en proceso de certificación de un modelo de calidad, muestran buenos resultados en su diagnóstico; y

3) El modelo de diagnóstico PSP es flexible para su mejora permitiendo aumentar su precisión. 


\section{REFERENCIAS}

Bertalanffy, L. V. Teoría General de los Sistemas. 7a reimpresión 1989, 311 págs., Fondo de Cultura Económica, México, (1968).

Cardona, S. A., Rincón, R. D., y Osorio, L. V., Implementación del Proceso Personal Software en un primer curso de Programación de Computadores, (2012).

Capra, F. La trama de la vida. Barcelona: Editorial Anagrama, (1998).

Chiavenato, I. Introducción a la teoría general de la administración. Santafé de Bogotá: McGraw-Hill, (1999). Chrissis, M. B., Konrad, M., y Shrum, S., CMMI for Development: Guidelines for Process Integration and Product Improvement. Addison-Wesley Professional, (2011).

Fernández, N., Investigación y técnicas de mercado. Editorial Esic, (2004).

Humphrey, W. S., Using a defined and measured personal software process. Software, IEEE, 13(3), 77-88, (1996).

Humphrey, W. S., Introducción al Proceso Software Personal. Primera Edición. PEARSON EDUCATION, S.A., Madrid, (2001).

Humphrey, W. S., Psp (sm): a self-improvement process for software engineers. Addison-Wesley Professional, (2005).

Johnson, P. M., y Disney, A. M. A critical analysis of PSP data quality: Results from a case study. Empirical Software Engineering, 4(4), 317-349, (1999).

Kampenes, V. B., Dybå, T., Hannay, J. E., Sjøberg, D. I., A systematic review of quasi-experiments in software engineering, Information and Software Technology, Volume 51, Issue 1, Pages 71-82, ISSN 09505849, (2009).

Kemerer, C. F., y Paulk, M. C. The impact of design and code reviews on software quality: An empirical study based on PSP data. Software Engineering, IEEE Transactions on, 35(4), 534-550, (2009).

Kitchenham, B. A., Pfleeger, S. L., Pickard, L.M., Jones, P. W., Hoaglin D. C., Emam K.E., and Rosenberg J. Preliminary Guidelines for Empirical Research in Software Engineering. IEEE Transactions on Software Engineering, (28)8, (2002).

Lee, T., Baik, D., y In, H. P., Cost benefit analysis of personal software process training program. In Computer and Information Technology Workshops, 2008. CIT Workshops 2008. IEEE 8th International Conference on (pp. 631-636). IEEE, (2008, July).

López, Y., André, M., y Infante, A. L., Formación de roles y buenas prácticas en el trabajo por la calidad de un ingeniero informático. Ingeniare. Revista chilena de ingeniería, 19(3), 382-395, (2011).

Morisio, M., Applying the PSP in Industry. Software, IEEE, 17(6), 90-95, (2000).

Robert L. C., Putting the Personal Software Process SM Into Practice. 12th Conference on Software Engineering Education and Training on (pp. 34-37).IEEE, (1999).

Salinas, E., Cerpa, N., y Rojas, P. Arquitectura orientada a servicios para software de apoyo para el proceso personal de software. Ingeniare. Revista chilena de ingeniería, 19(1), 40-52, (2011).

Seaman, C. B., Qualitative methods in empirical studies of software engineering. Software Engineering, IEEE Transactions on, 25(4), 557-572, (1999).

Silberberg, D., Applying the personal software process (PSP) with Ada. In ACM SIGAda Ada Letters (Vol. 18, No. 6, pp. 219-228). ACM, (1998).

Silliti, A., Janes, A., Succi, G., Vernazza, T. Collecting, Integrating and Analyzing Software Metrics and Personal Software Process Data. In Proceedings of the 29th EUROMICRO Conference "New Waves in System Architecture". IEEE, (2003). 
Sison, R., Personal software process (psp) assistant. In Software Engineering Conference, 2005. APSEC'05. 12th Asia-Pacific (pp. 8-pp). IEEE, (2005).

Syu, I., Salimi, A., Towbidnejad, M., y Hilburn, T., A web-based system for automating a disciplined personal software process (PSP). In Software Engineering Education and Training. Tenth Conference on (pp. 86-96). IEEE, (1997).

Vallejo, P. M., Tamaño necesario de la muestra,http://www.upcomillas.es/personal/peter/investigacion/Tama \% F1omuestra.pdf. Acceso: 2 de Noviembre (2012)

Wohlin, C., y Wesslén, A., Understanding software defect detection in the personal software process. In Software Reliability Engineering, 1998. Proceedings. The Ninth International Symposium on (pp. 49-58). IEEE, (1998). 